\title{
Microbiology Susceptibility Result Standard Unit
}

National Cancer Institute

\section{Source}

National Cancer Institute. Microbiology Susceptibility Result Standard Unit. NCI

Thesaurus. Code C87940.

The standard unit of measure for microbiology susceptibility results. 